\title{
A Modular Folded Laminate Robot Capable of Multi Modal Locomotion
}

\author{
Je-sung Koh ${ }^{1}$, Daniel M. Aukes ${ }^{1,2}$, Brandon Araki ${ }^{3}$, Sarah Pohorecky ${ }^{3}$, \\ Yash Mulgaonkar ${ }^{4}$, Michael T. Tolley ${ }^{5}$, Vijay Kumar ${ }^{4}$, \\ Daniela Rus ${ }^{3}$, and Robert J. Wood ${ }^{1}$ \\ ${ }^{1}$ John A. Paulson School of Engineering and Applied Sciences and the Wyss \\ Institute for Biologically Inspired Engineering, Harvard University. \\ ${ }^{2}$ The Polytechnic School, Fulton Schools of Engineering, Arizona State University. \\ ${ }^{3}$ CSAIL, The Stata Center, Massachusetts Institute of Technology. \\ 4 The GRASP Laboratory, University of Pennsylvania. \\ ${ }^{5}$ Dept. of Mechanical and Aerospace Engineering, University of California, San \\ Diego.
}

\begin{abstract}
This paper describes fundamental principles for two - dimensional pattern design of folded robots, specifically mobile robots consisting of closed-loop kinematic linkage mechanisms. Three fundamental methods for designing closed-chain folded four-bar linkages - the basic building block of these devices - are introduced. Modular connection strategies are also introduced as a method to overcome the challenges of designing assemblies of linkages from a two-dimensional sheet. The result is a design process that explores the tradeoffs between the complexity of linkage fabrication and also allows the designer combine multiple functions or modes of locomotion. A redesigned modular robot capable of multi-modal locomotion and grasping is presented to embody these design principles.
\end{abstract}

Keywords: Printable robots, Folded laminate devices, Multi-modal locomotion

\section{Motivation, Problem Statement and Related Work}

Folding-based mechanisms inspired by origami have recently become popular for rapid design iterations for complex structures and mechanisms. These methods are low-cost and inherently accessible, providing non-experts access to robot design [1]. In addition, the scalability of folding-based concepts enables new ways to create robots over size scales from micro to meso scales [2-4]. The potential of folding as an assembly method has been demonstrated in education [5] and in research exploring biological hypotheses in bio-mimetic robots [6-8].

One of the primary advantages of folded robot design is that the inherent accessibility and low cost of the method permits designers to get design feedback early and often via fast prototyping cycles. While prototyping is relatively fast, the complexity of multi-layer articulated designs can be time-consuming and 
unwieldy to design; efforts have recently been made to develop computer-aided design software and an integrative design methodology to make the design and manufacture of foldable robots more convenient for non-experts $[9,10]$. Consequently, the development environment for the design and fabrication of foldingbased mechanisms has advanced significantly. Manufacturing principles for laminates [10] and design principles for folding [11] have been abstracted mathematically, permitting the automated merging of complex fold patterns [12,13], and advancing the development of various innovative fabrication methods, such as self-folding [14] and printed-circuit MEMS (PC-MEMS) [15].

In this paper, we present an intuitive design methodology to map a 3D kinematic linkage mechanism to a 2D fold pattern design. The robot design starts with drawing lines and points to specify an abstract linkage mechanism, as in conventional machine design; however, the lines and points are then transferred to a 2D folding pattern by using one of three fundamental methods. A folded laminate crawler is then built by using the proposed method. Experiments on the crawler show the inherent compliance of a folding-based robot. A modular design concept for the folded robot is described to reduce design complexity and enhance convenience in combining more than two functions or modes of locomotion. This work builds on the previous "Flying Monkey" robot [16] by a simplifies the design to a single folded sheet and introduces modularity of components and appendages to streamline the customization of developing a low-cost multi-modal robot.

\section{Folding Pattern Design}

Robots, particularly legged robots, often have kinematic linkages composed of links and various types of mechanical joints that map an actuator or motor input to a desired motion. The mechanism can be described by lines and points that correspond to links and joints in an analogous way to the axes of rotation and coordinate frames of the Devavit-Hartenberg convention. In mechanism design, designers generally start with drawing lines and points to create an abstract schematic diagram of the desired linkage. After drawing the schematic diagram, a type of joint, such as a revolute, prismatic, or spherical joint, is defined at each point to complete a mechanism. This step determines the kinematics of the mechanism, such as the range of motion and degrees of freedom (DOF). In converting this abstract schematic to a $2 \mathrm{D}$ fold pattern, revolute joints that correspond to pin or hinge joints in conventional mechanical designs are transformed to fold lines connecting two facets with a single degree of freedom. The fold line and the facet are the basic elements of the folding mechanism. The pattern design consists of placing these basic elements at links and joints of the schematic diagram on a 2-dimensional plane that allows them to be folded into the three-dimensional mechanism. These two corresponding elements in folding pattern design and mechanical design - the fold line and the revolute joint - enable a designer to transform a schematic diagram into a folding pattern design intuitively. As the complexity of a mechanism increases, however, the pattern 

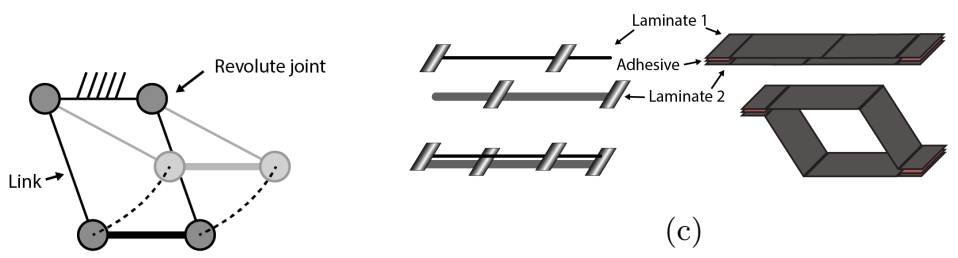

(c)

(a)

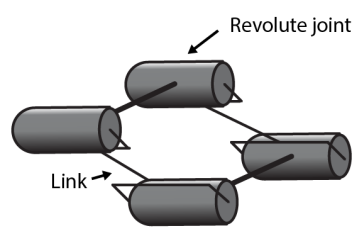

(b)

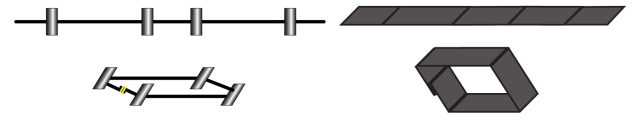

(d)
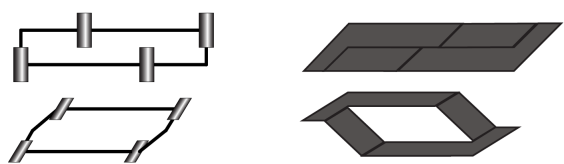

(e)

Fig. 1: Pattern designs for a four-bar mechanism. (a) Schematic diagram of a four-bar mechanism and its range of motion. (b) The four-bar mechanism consists of revolute joints and links in three dimensions for conventional machine design. (c) The multi-laminate (d) joining two ends of a single serial link and (e) the joint offset design.

design becomes non-intuitive, and the resulting folded shape and motion of the mechanism become difficult to visualize.

\section{Technical Approach and Results}

\subsection{Fold Pattern Design for Closed Chain linkages}

Most linkages consist of closed chains that generate a desired motion from actuators which have a limited range of motion. The design of closed (parallel) chains can often be more challenging than for open (serial) chains, due to the additional constraints imposed by flat laminate fabrication methods. A closed mechanism assembled in its flat state loses design freedom by the fact that link vectors must sum to zero during planar fabrication steps. Here we propose three classes of intuitive approaches to designing closed-chain folding mechanism; these approaches can be applied to general folding pattern design with this known tradeoff.

To illustrate the application of the three design methods, we will describe how they can be applied to the folding pattern design of a four-bar linkage mechanism. A four-bar linkage mechanism is a simple, single-degree-of-freedom planar mechanism with few canonical design variables, often employed in robotic 


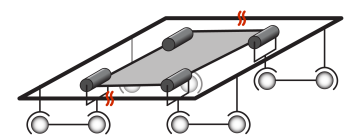

(a)

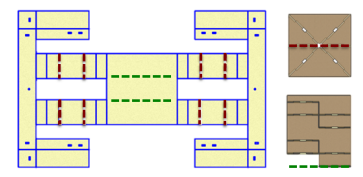

(d)

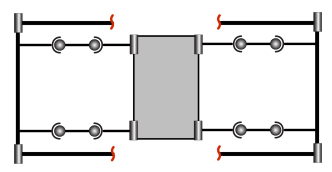

(b)

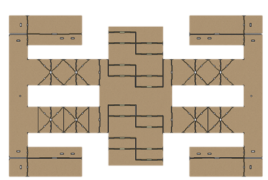

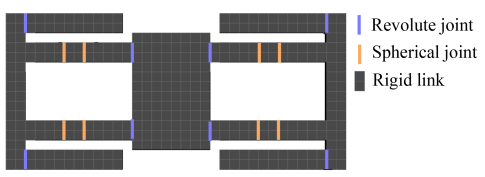

(c)

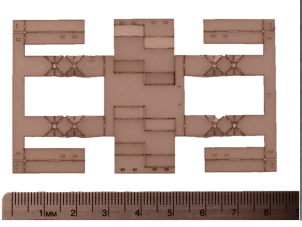

(e)

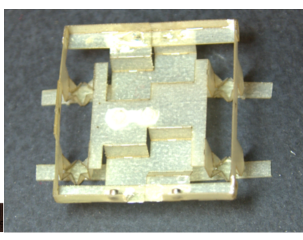

(f)

Fig. 2: (a) Kinematic diagram for the crawler with four legs. (b) Flattened mechanism after cutting the exterior link. (c) Transforming from lines and points to facets and fold lines. (d) Generating detailed cut lines by placing 2D patterns of mechanical elements. (e) The single foldable laminate for the crawler. (f) Folded laminate crawler after assembly

applications $[4,15]$. Four-bar linkages with equal-length opposite links maintain parallelism between these links while permitting rotation of the interior angles, as shown in fig.1a, and are often used as transmissions to transform linear reciprocating motion into rotational motion or vice-versa. As in fig.1b, this can be depicted in an abstract three dimensional perspective. In a two-dimensional design, however, a significantly different approach to draw this kinematic diagram is needed because it is derived from flat surfaces. The first method consists of stacking and joining layers, which is called the "multi-laminates" method as shown in fig. 1c. By joining facets at each end of the structure, the folding mechanism becomes a closed chain when folded as depicted in the right side of fig.1c. The second method requires joining the ends of a single serial link as shown in fig. 1d. This permits individual links to be of any arbitrary length, however a manual assembly step is required to join the two ends before folding. In addition, a much larger machining area (on a single layer) is used compared to the first method. These two methods can be designed intuitively. However, the third strategy - the "joint offset" method - can be less intuitive. In this case, links are arranged beside each other (rather than on top of each other) in order to permit links to be cut from a single layer of material. In other words, links which would normally overlap are shifted along their rotational axes until they are neighboring facets in a single sheet of material, as shown in fig.1e. This method allows the 2D pattern design of the closed chain to be designed in a single plane without post-processing. Almost all kinematic linkage mechanisms can be designed by 

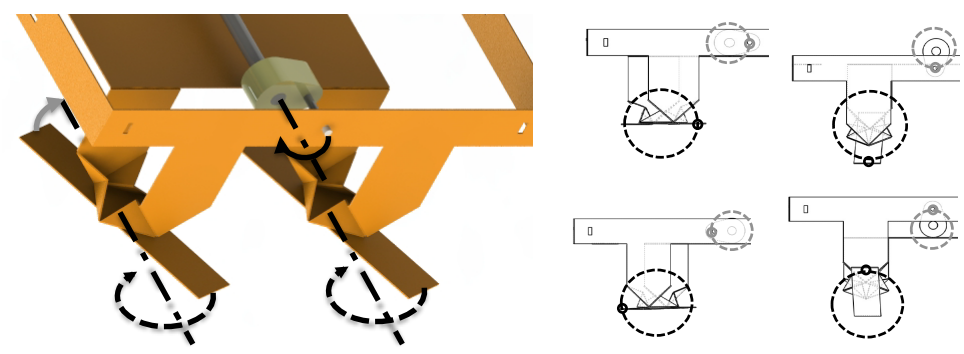

Fig. 3: Leg motion of the folded laminate crawler.

a combination of these three methods, although the angle and length of links allow variation in the shape of a folding pattern.

\subsection{Single Sheet Crawler Design}

To make a multi-legged crawling robot, we designed a closed chain linkage that has two primary links - interior and exterior frames - which are linked by four serial linkages as shown in fig.2a. This type linkage has been the basis of many previous crawling robots that were built with folded laminate mechanisms $[3,4$, 16]. In this paper, two spherical joints are employed in four serial leg links to generate the rotational motion of feet that will be attached under the middle link. The motor rotates between the interior link and the exterior link with a crank shaft as shown in fig. 3 . For patterning on a single sheet, the exterior linkage is cut and the linkage is deployed and flattened on a plane as shown in fig.2b. After fabrication of the folding laminate, the robot mechanism is finished by joining the exterior linkage. The task of joining (i.e. gluing) is simple and intuitive but it becomes onerous in complex mechanisms. Currently, many technique can be applied such as a solder fillet locking [17], a shape memory alloy riveting [18].

After sketching the mechanism diagram, links and joints are transformed into facets and fold lines intuitively, as shown in fig. 2c, and the detailed shape of the fold pattern is determined. The square shape is the basic unit cell, because all links and joints are perpendicular to each other in this case, but other kinds of polygons can be used, as a unit cell depends on the relative direction of joints and links. The spherical joints are marked as single lines at this step, but they will be replaced by a spherical six-bar pattern which has three DOF (pitch, roll, and yaw) motion between two opposite links [19].

\subsection{Fabrication}

Given the desired 2D patterns for the linkage elements, the detailed cut patterns can be drawn using computer-aided design (CAD) software. PopupCAD [20] has been developed as a convenient user interface for designing the folded-laminate devices. The kinematic pattern design (fig. 2c) is drawn in Solidworks (Dassault 
Systems Co.). By importing the design into PopupCAD, the detailed 2D pattern elements are placed in corresponding locations as shown in fig. $2 \mathrm{~d}$. In the crawler design, the spherical joints and four-bar linkages are added in the kinematic mechanism. The spherical six-bar linkages are placed as spherical joints that have three DOFs. In addition, a serial four-bar linkage as shown in fig. $2 \mathrm{~d}$ is added at the green dotted line in the main mechanism to constrain the final mechanism to 2-DOF rotational motion. The 2D pattern design of the serial four-bar linkage is a serial connection of two four-bar linkages that is drawn by the joint-offset method described in fig.1e. Each end of the serial four-bar linkage is attached at the interior and the exterior link, and the relative motion between the two links is constrained into parallel circular motion.

The manufacturing process for the folding laminate is Printed-Circuit MEMS (PC-MEMS) (Fig. 2e-2f) [10,15]. The detailed cut lines for film layers are generated by PopupCAD automatically while verifying the manufacturability of each layer. The folding laminate in this paper has five layers: a rigid layer, an adhesive layer, a flexure layer, an adhesive layer, and a rigid layer. The right-hand side of fig. $2 \mathrm{~d}$ is the final five-layer design of the crawler. Each layer is cut by laser machining and laminated by a thermal laminator. After the initial cut, each layer has alignment pin holes in support parts which are cut out during the final cut. Fig. 2e shows the laminate after the final cut. The laminate is assembled by joining the exterior link and each end of serial four-bar linkages as shown in fig. 2f. Finally, the four legs, are attached; the legs can be changed to suit the terrain the robot will be crawling over. The legs can be added in the laminate design but separated in order to test various leg designs easily.

\subsection{The Folded Laminate Crawler}

The crawler is driven by a DC motor with a crank shaft. The motor is mounted on the interior link and the crank shaft is connected to the exterior link. The interior link and the exterior link rotate with respect to each other and the leg links between the two frames swing in a rowing motion as shown in fig. 3. In this case, the rotation radius of the crank shaft is $1.5 \mathrm{~mm}$, the length of the leg

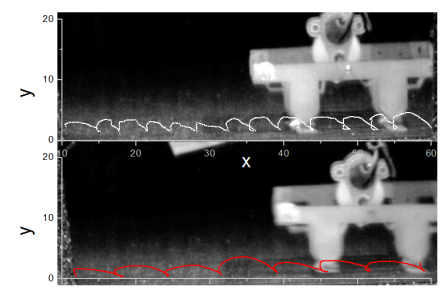

(a)

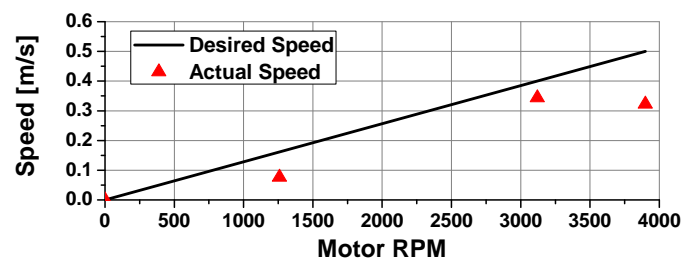

(b)

Fig. 4: (a) Plot tracking the foot at $21 \mathrm{~Hz}$ (upper) and $52 \mathrm{~Hz}$ (lower). (b) Crawling speed versus motor driving speed. 
Table 1: Performance of The crawler

\begin{tabular}{r||ccc} 
& \multicolumn{4}{|c}{} & $21 \mathrm{~Hz}$ & $52 \mathrm{~Hz}$ & $65 \mathrm{~Hz}$ \\
\hline Designed speed [m/s] & 1.6 & 4 & 5 \\
Experimental speed [m/s] & 0.7 & 3.4 & 3.2 \\
Designed stride length [mm] & 7.7 & 7.7 & 7.7 \\
Experiemtnal stride length [mm] & 3.6 & 6.6 & 5
\end{tabular}

link is $6.5 \mathrm{~mm}$, and the legs are $18.5 \mathrm{~mm}$. By kinematic calculation, the stride length and the swing angle of the legs are $7.7 \mathrm{~mm}$ and 25 degrees. However, the performance of the crawler varies depending on the driving speed (i.e., rotational frequency of motor). As the driving frequency increases, both speed and stride length increase as shown in fig. 4. However, unfortunately, the crawler starts to flip over in high frequencies above $52 \mathrm{~Hz}$ and the speed and the stride length drop at $62 \mathrm{~Hz}$. Experimental results are listed in Table. 1.

Folded laminate devices are inherently compliant because the links are sheets which have a low bending stiffness induced by a low area moment of inertia. Therefore, the speed and the stride length changes based on the driving frequency. Fig. 5 shows the tracking data and its frequency spectrum for the crank shaft and the foot driving in air. The foot is more sensitive to driving frequency than the crank shaft, and the range of fluctuation in vertical motion is much larger than the horizontal motion. This may be caused by the limited horizontal range of motion in the spherical six-bar linkages which are used as the spherical joints. The compliance of the structure may reduce precision and linearity of the system and cause difficulties in control.

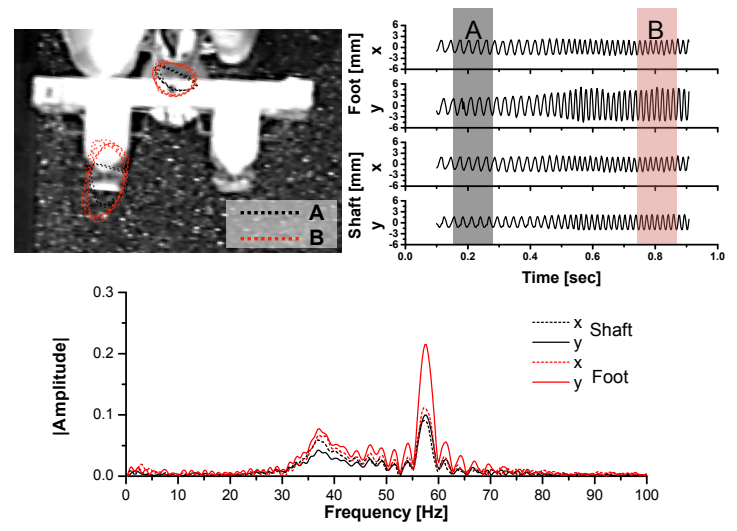

Fig. 5: Trajectory of the shaft and the foot in air (upper) and frequency response of the trajectories (lower). 


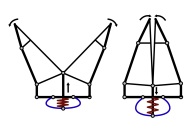

(a)

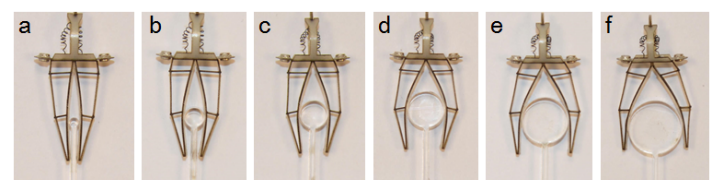

(d)

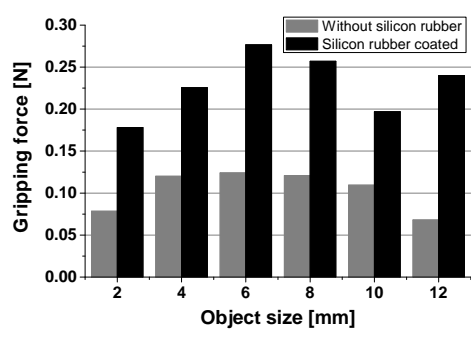

(c)

Fig. 6: (a) Kinematic diagram for the adaptive gripper. (b) 2D pattern design for the gripper. (c,d) Gripping force experiments demonstrating adaptive gripping in various size of objects form $2 \mathrm{~mm}$ to $12 \mathrm{~mm}$ in diameters (a-f, $2 \mathrm{~mm}$ increments).

\subsection{Adaptive Gripper}

The gripper design is adapted from the Festo adaptive gripper [21]. To decrease the size of the gripper, the minimum number of links are used, as in the kinematic diagram in fig. 6a. Using the pattern design method describe in section. II, the kinematic linkage is flattened and transformed into a $2 \mathrm{D}$ pattern as shown in fig. 6b. The closed linkage can be made by joining each end of the serial links; the links inside of the closed link are positioned by an offset joint in the middle of facets.

The gripper is opened by a coiled shape memory alloy actuator placed at the middle links as indicated by the red coil in fig.6a and closed by the passive plate spring indicated by the blue curved links in fig. $6 \mathrm{a}$ and two blue facets in fig. $6 \mathrm{~b}$. Therefore, the gripper grips an object passively using the spring, so that it is not required to apply a force to hold the object. The adaptive gripper grasps the object by bending two internal links as shown in fig. 6d. Each segment of fingers is zero-DOF. The compliance of the sheet link enables adaptable gripping with various size of objects as shown in fig. $6 \mathrm{c}$.

\subsection{Modularization with Magnetic Connections}

Due to the limited work space for the 2D pattern design and complexity caused by having multiple components in a single multi-functional device, a modularized design is a simpler and more effective way to design folded laminate devices. The system can be modularized into mechanisms, motors, accessories, and interfaces in a high level design. Each module can be designed separately but with a common connection/mounting protocol that allows the modules to be combined and recombined very easily as described in fig. 7 . In this paper, a gripper module and a crawler module are described and assembled with a magnetic mount which is capable of automatic positioning and provides electric power connections. In 


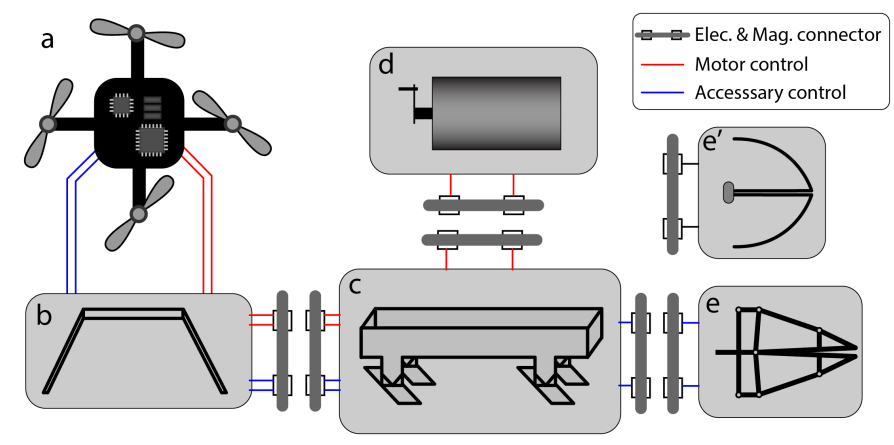

Fig. 7: Schematic diagram of the modular multi-modal robot assembled by magnetic connectors for mechanical and electrical connection. a: quadrotor, b: magnetic mount for the quadrotor, c: crawler, d: motor, e: a gripper accessory, e': antenna accessory.

addition, motors and other interfaces such as to connect to a quad-rotor can be modularized for pick-and-place functionality.

In contrast to the philosophy of origami, in which shapes are folded from a single sheet of paper, a multi-laminate and modularized design may reduce the complexity of a multifunctional robot by making it easier to join components together while also expanding the design space and feasible functions of the foldable robot. As shown in fig. 8, the adaptive gripper accessory module, the crawler interface module, and the motor modules are manufactured separately. They have magnetic mounts whose poles are synchronized with each other. Red dotted lines circle the magnetic mount for the gripper and black dotted lines circle the magnetic mount for the motor. When the modules are placed close to their positions, the magnets pull each other and attach to their mounts (forming both electrical and mechanical connections) automatically. Two pairs of Neodymium magnets (2 $\mathrm{mm}$ in diameter, $1.6 \mathrm{~mm}$ in thickness) are used for the magnetic mount and the pulling load is $180 \mathrm{~g}$ each and $360 \mathrm{~g}$ as a pair. The magnetic force is much higher than the crawler's weight $(4.5 \mathrm{~g})$ and it is hard to break the connection while it moves.

To be capable of multiple functions and multi-modal locomotion, various accessory and interface modules can be attached by pick-and-place. As an accessory module, the active antenna shown in fig. 8 (d) steers the crawler by changing its center of mass. As an interface module for multimodal locomotion, a small quad-rotor, an open-source drone commercially available (Crazyflie 2.0 [22]), can drastically improve the range and speed of the robot as shown in fig. 8 .

\section{Results}

Intuitive folding patterns are directly transformed from the kinematic linkage diagrams that are used as conceptual designs in conventional machine design. In 

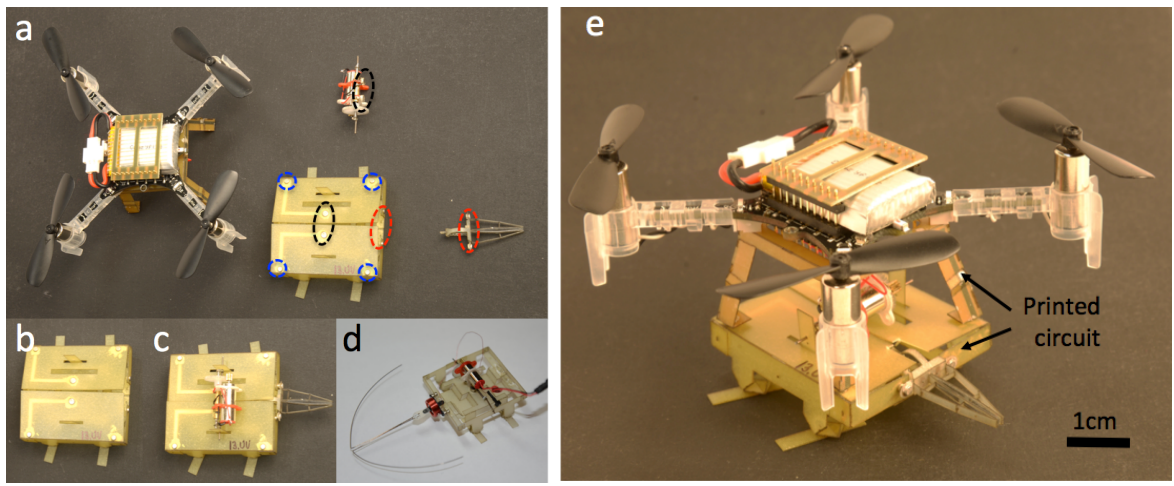

Fig. 8: Modular folded laminate crawler. (a) Gripper accessory module, crawler interface module, motor module with magnetic mounts (dotted lines) and (b) Crawler with the motor module. (c) Crawler with the motor and the gripper modules and (d) the antenna module. (e) Crawler with the gripper accessory and the quad-rotor interface module for multi-modal locomotion, flying, gripping, and crawling.

this paper, a folded laminate crawler and a adaptive gripper are designed on a single sheet using three fundamental methods for designing a closed chain linkage mechanism in 2D space. The inherent compliance of the folding structure causes the folded laminate crawler to exhibit resonant behavior. The concept of modular design may reduce complexity in the folding pattern design by modularization of interfaces for multimodal locomotion, accessories for additional functions, and actuators.

To demonstrate multi-modal functionality, the robot performs specific tasks by flying, gripping, and crawling. To reduce complexity of modules in terms of the number of actuators, each module has a single actuator which needs just a single control and power input. For this reason, crawler can crawl only forward and not steer. However, the quadrotor can introduce additional torques, so that the robot can be controlled by the microcontroller on quadrotor. Figure 9 shows the robot crawling straight, gripping, and hovering controlled by the quadrotor in a motion capture arena. A similar ground locomotion control scheme was employed by the original Flying Monkey [16].

\section{Discussion}

To simplify the design and fabrication of robots, foldable design methods must be further abstracted and automated. The design methods presented in this paper may not be applicable to specific cases such as a robot capable of transporting high payloads. Static and kinematic requirements should be addressed in design, for example by inserting extra folding links to improve the robustness of linkages based on analytical or numerical models of the device. Lastly, the selection of 


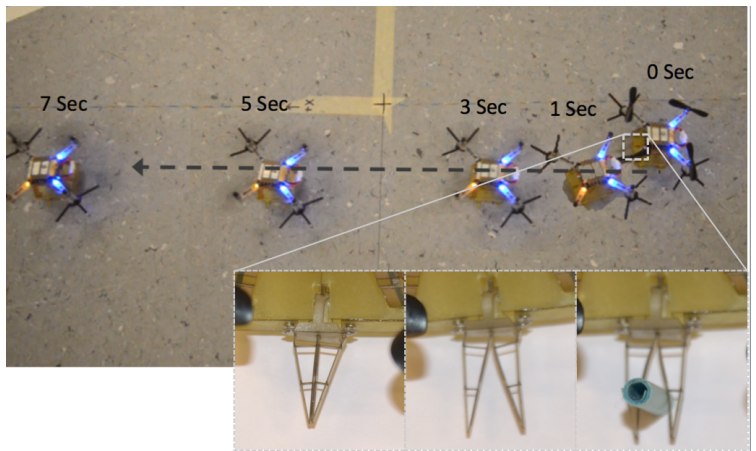

(a)

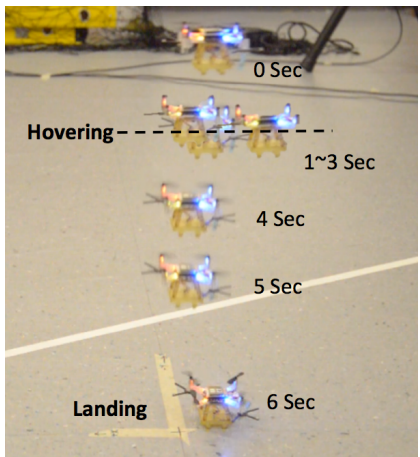

(b)

Fig. 9: The Flying Monkey robot moves with a feedback control in a motion capture arena.(a) Controlled crawling and gripping. The robot following the straight line (dotted-line). (b) Controlled flight.

proper actuation and power delivery methods for modular robots is required to finalize the robotic system for operation in the field.

\section{ACKNOWLEDGMENT}

This research was supported by the National Science Foundation (EFRI-1240383 and CCF- 1138967) and and the Wyss Institute for Biologically Inspired Research. Any opinions, findings, and conclusions or recommendations expressed in this material are those of the authors and do not necessarily reflect those of the National Science Foundation.

\section{References}

1. Bezzo, N., Mehta, A., Onal, C.D., Tolley, M.T.: Robot makers: The future of digital rapid design and fabrication of robots. IEEE Robotics Automation Magazine 22(4) 27-36 (2015)

2. Wood, R., Avadhanula, S., Sahai, R., Steltz, E., Fearing, R.: Microrobot design using ber reinforced composites. Journal of Mechanical Design, 130(5), 052304 (2008)

3. Hoover, A.M., Steltz, E., Fearing, R.S.: Roach: An autonomous 2.4g crawling hexapod robot. In: Intelligent Robots and Systems, 2008. IROS 2008. IEEE/RSJ International Conference on. , 26-33 (2008)

4. Birkmeyer, P., Peterson, K., Fearing, R.S.: Dash: A dynamic 16g hexapedal robot. Intelligent Robots and Systems, 2009. IROS 2009. IEEE/RSJ International Conference on, IEEE 2683-2689 (2009)

5. Cybulski, J.S., Clements, J., Prakash, M.: Foldscope: Origami-based paper microscope. PLoS ONE 9(6) 1-11 (2014)

6. Ma, K.Y., Chirarattananon, P., Fuller, S.B., Wood, R.J.: Controlled fight of a biologically inspired, insect-scale robot. Science 340(6132) 603-607 (2013) 
7. Koh, J.S., Yang, E., Jung, G.P., Jung, S.P., Son, J.H., Lee, S.I., Jablonski, P.G.,Wood, R.J., Kim, H.Y., Cho, K.J.: Jumping on water: Surface tensiondominated jumping of water striders and robotic insects. Science $349(6247), 517-521$ (2015)

8. Jayaram, K., Full, R.J.: Cockroaches traverse crevices, crawl rapidly in connedspaces, and inspire a soft, legged robot. Proceedings of the National Academy of Sciences 113(8) E950-E957 (2016)

9. Mehta, A., DelPreto, J., Rus, D.: Integrated codesign of printable robots. Journal of Mechanisms and Robotics 7(2) 021015 (2015)

10. Aukes, D.M., Goldberg, B., Cutkosky, M.R., Wood, R.J.: An analytic framework for developing inherently-manufacturable pop-up laminate devices. Smart Materials and Structures 23(9) 094013 (2014)

11. Demaine, E.D., ORourke, J.: Geometric folding algorithms. Cambridge university press Cambridge (2007)

12. Sung, C., Demaine, E.D., Demaine, M.L., Rus, D.: Joining unfoldings of 3-d surfaces. In: ASME 2013 International Design Engineering Technical Conferences and Computers and Information in Engineering Conference, American Society of Mechanical Engineers V06BT07A033-V06BT07A033 (2013)

13. Onal, C., Tolley, M., Wood, R., Rus, D.: Origami-inspired printed robots. Mechatronics, IEEE/ASME Transactions on 20(5) 2214-2221 (2015)

14. Felton, S., Tolley, M., Demaine, E., Rus, D., Wood, R.: A method for building self-folding machines. Science 345(6197) 644-646 (2014)

15. Whitney, J.P., Sreetharan, P.S., Ma, K.Y., Wood, R.J.: Pop-up book mems. Journal of Micromechanics and Microengineering 21(11) 115021 (2011)

16. Mulgaonkar, Y., Araki, B., sung Koh, J., Guerrero, L., Aukes, D.M., Makineni, A.,Tolley, M.T., Rus, D., Wood, R.J., Kumar, V.: The Fying monkey, a multifunctional mesoscale robot that can run, and grasp. IEEE Int. Conference on Robotics and Automation(ICRA) (2016)

17. P S Sreetharan and J P Whitney and M D Strauss and R J Wood : Monolithic fabrication of millimeter-scale machines. Journal of Micromechanics and Microengineering 22(5), 055027 (2012)

18. Ji-Suk Kim, Dae-Young Lee, Je-Sung Koh, Gwang-Pil Jung and Kyu-Jin Cho.: Component assembly with shape memory polymer fastener for microrobots, Smart Materials and Structures 23(1), 015011 (2014)

19. Koh, J.S., Cho, K.J.: Omega-shaped inchworm-inspired crawling robot with largeindex-and-pitch (LIP) SMA spring actuators. IEEE/ASME Transactions on Mechatronics 18(2) 419-429 (2013)

20. Popupcad. http://www.popupcad.org/

21. Festo. https://www. festo.com/cms/encorp/9779.htm

22. Crazyflie 2.0 https://www. bitcraze.io/ 